\section{Field-screening Prunus for Longevity in the Southeastern United States}

\author{
W.R. Okie \\ U.S. Department of Agriculture, Agricultural Research Service, Southeastern \\ Fruit and Tree Nut Research Laboratory, P.O. Box 87, Byron, GA 31008
}

G.L. Reighard

Department of Horticulture, Clemson University, Clemson, SC 29634

T.G. Beckman, A.P. Nyczepir, and C.C. Reilly

U.S. Department of Agriculture, Agricultural Research Service, Southeastern Fruit and Tree Nut Research Laboratory, P. O. Box 87, Byron, GA 31008

E.I. Zehr and W.C. Newall, Jr.

Department of Plant Pathology, Clemson University, Clemson, SC 29634

D.W. Cain

Sun World International, P. O. Box 80298, Bakersfield, CA 93380

Additional index words. peach, plum, peach tree short life, rootstock, replant

Abstract. Long-term field trials of a wide range of peach [Prunus persica (L.) Batsch] germplasm on two peach tree short-life (PTSL) sites revealed marked differences in survival among lines. Generally, cuttings and seedlings of a given line performed similarly, as did ungrafted seedlings and their counterparts grafted to a commercial cultivar. No apparent relationship existed between a line's chilling requirement and survival. B594520-9 survived best in Georgia and South Carolina, providing significantly greater longevity than Lovell, the standard rootstock for use on PTSL sites. B594520-9 is derived from root-knot-nematode-resistant parentage, and progeny of surviving seedlings have demonstrated root-knot resistance similar to Nemaguard seedlings.

The productive life of peach trees in the southeastern United States is reduced by the syndrome known as peach tree short life (PTSL). Affected trees suddenly collapse and die in the spring, usually 3 to 6 years after planting (Ritchie and Clayton, 1981). The disease complex results from an interaction of weather, ring nematode [Criconemella xenoplax (Raski) Luc \& Raski] parasitism, and cultural practices, such as previous crop, rootstock, and pruning date (Sharpe et al., 1989). Scion death is apparently caused by damage to the trunk from either cold injury or bacterial canker (Pseudomonas syringae pv. syringae van Hall). Chemical control of $\mathrm{C}$. xenoplax, and hence PTSL, is currently possible with annual postplant nematicides, but such control is often not cost-effective. Land with no recent history of peach plantings is becoming scarce and expensive; it often has a microclimate marginally suitable for reliable peach production. Currently, Lovell peach rootstock is recommended to maximize tree longevity on PTSL sites.

In field plots and greenhouse pot tests, Lovell often supports lower ring nematode populations than Nemaguard, but both are good hosts (Okie et al., 1987; Sharpe et al.,

Received for publication 26 May 1993. Accepted for publication 28 Sept. 1993. The cost of publishing this paper was defrayed in part by the payment of page charges. Under postal regulations, this paper therefore must be hereby markedadvertisement solely to indicate this fact. greenhouse tests use less space and time than field tests, reduced host suitability may not
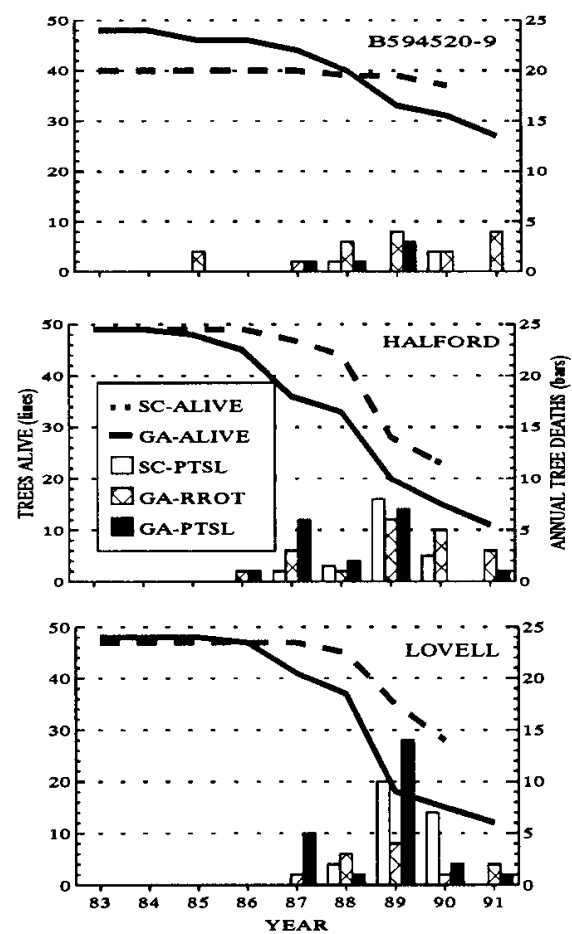

translate into longer life in the orchard. Therefore, field screening for longevity remains the definitive test for rootstock selection.

Due to the time and effort involved in field screening for longevity, only a few attempts have been made to identify long-lived Prunus germplasm. A few selections were made by C.N. Clayton and F.E. Correll in North Carolina about 40 years ago (unpublished). F.E. Johnstone, Univ. of Georgia, also made a modest effort in the late 1960s to collect longlived local selections (John stone, 1971), but the program was short-lived. S. Doud, Fort Valley State College, continued this work at Byron for a few years in the late 1970s.

Lovell is susceptible to root-knot nematodes (Meloidogyne spp.). Where this nematode is common, Nemaguard rootstock is recommended, although trees on Nemaguard die quickly on PTSL sites. None of the other rootknot-resistant rootstock tested in the past, such as Yunnan, Shalil, and S-37, have survived well on PTSL sites in the southeastern United States. A rootstock conferring longer tree life, particularly in combination with rootknot-nematode resistance, would be of great value to the industry and is the primary goal of this research project. Preliminary reports of this work have been published (Cain et al., 1986; Okie et al., 1991; Reighard et al., 1989a, 1989b).

\section{Materials and Methods}

Open-pollinated seed of $>140$ diverse accessions of peach and plum (diploid Prunus hybrids) were collected in 1982, mostly at Byron, Ga., and planted in a nursery at Clemson, S.C. Unbudded trees were tested because of
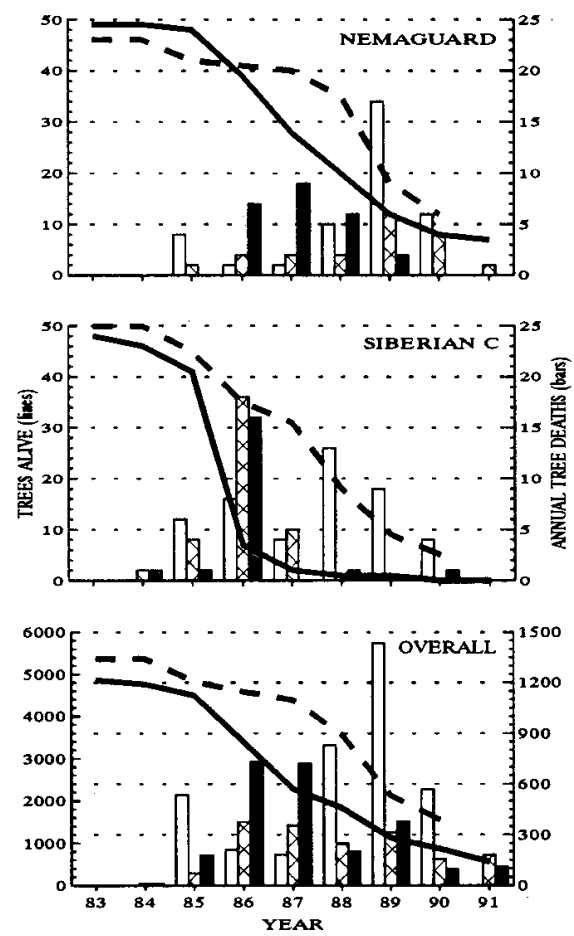

Fig. 1. Number of trees still alive (left axis and lines) and annual tree losses (right axis and bars) by cause overall and for seedlings of selected peach rootstocks. Field screening conducted in Byron, Ga., and Elgin, S.C., 1983-91. All South Carolina deaths due to peach tree short life (PTSL). Some Georgia mortality also caused by Clitocybe root rot (RROT). 
Table 1. Accession type, total number of trees $(\mathrm{N})$, and percent tree death due to peach tree short life (PTSL) for peach and plum accessions screened at Byron, Ga., and Elgin, S.C., 1983-91. Georgia percentage also is expressed using data set that excludes any trees that died of Clitocybe root rot.

\begin{tabular}{|c|c|c|c|c|c|c|c|c|c|c|}
\hline & & & & & & outh & & Georg & & Both \\
\hline & ccession & & & & & rolina & & & SL & states \\
\hline NA/PI & RK/RS & $\mathrm{PL}$ & $\overline{\mathrm{CV}}$ & Accession & $\mathrm{N}$ & $\%$ PTSL & $\mathrm{N}$ & All trees & $-{\text { Root } \operatorname{rot}^{y}}^{y}$ & $\%$ PTSL \\
\hline & RK & & & B594520-9 & 40 & 7.5 & 48 & 10.5 & 17.1 & 9.0 \\
\hline & RS & & & B14DR51 & 47 & 23.4 & 47 & 21.2 & 37.1 & 22.3 \\
\hline NA & & & & J68-271 & 47 & 23.4 & 48 & 29.1 & 76.6 & 26.3 \\
\hline & & PL & & Edible Sloe & 43 & 44.1 & 14 & 11.3 & 20.0 & 27.7 \\
\hline & RK & & & B594520-8 & 42 & 21.4 & 49 & 37.1 & 64.2 & 29.3 \\
\hline & & PL & & BY7789-303 & 0 & --- & 14 & 33.2 & 55.6 & 33.2 \\
\hline & & PL & & BY7446-14 & 41 & 53.6 & 42 & 23.8 & 30.7 & 38.7 \\
\hline NA & RS & & & J67-34 = Tennessee Natural OP & 48 & 33.3 & 42 & 45.1 & 80.6 & 39.2 \\
\hline NA & RS & & & Tennessee Natural R4 & 48 & 54.1 & 48 & 27.1 & 75.0 & 40.6 \\
\hline & RS & & & B14DR52 & 46 & 50.0 & 33 & 32.1 & 100 & 41.1 \\
\hline & RK & & & Nemaguard cutting & 22 & 45.4 & 48 & 37.5 & 67.5 & 41.5 \\
\hline & & PL & & Blue Goose & 35 & 62.8 & 29 & 20.8 & 36.0 & 41.8 \\
\hline & RS & & $\mathrm{CV}$ & Lovell cutting & 48 & 56.2 & 47 & 29.1 & 53.2 & 42.7 \\
\hline PI & & & $\mathrm{CV}$ & Transvaal Yellow & 44 & 40.9 & 50 & 45.2 & 54.5 & 43.1 \\
\hline NA & & & & SCRS3 & 47 & 53.1 & 48 & 33.5 & 52.4 & 43.3 \\
\hline NA & & & & GA102 = Indian Cling & 46 & 50.0 & 48 & 37.5 & 69.3 & 43.8 \\
\hline & RS & & $\mathrm{CV}$ & Lovell VF & 48 & 40.4 & 48 & 48.0 & 67.8 & 44.2 \\
\hline & RS & & $\mathrm{CV}$ & Halford & 49 & 53.0 & 49 & 35.5 & 57.8 & 44.3 \\
\hline PI & RK & & & J67-14 = Okinawa OP & 47 & 46.8 & 49 & 42.1 & 86.4 & 44.5 \\
\hline PI & & & $\mathrm{CV}$ & Baladi & 44 & 47.7 & 48 & 43.8 & 61.2 & 45.8 \\
\hline & & & $\mathrm{CV}$ & Pekin/Lovell & 46 & 60.8 & 48 & 31.3 & 52.5 & 46.1 \\
\hline NA & RS. & & & NA2 & 47 & 55.3 & 48 & 39.5 & 70.7 & 47.4 \\
\hline & RK & & & $\mathrm{NC} 152-\mathrm{AI}-2 \mathrm{RH}=\mathrm{S}-37 \mathrm{OP}$ & 43 & 55.8 & 48 & 39.6 & 65.1 & 47.7 \\
\hline & RS & & $\mathrm{CV}$ & Halford cutting & 38 & 60.6 & 48 & 35.5 & 71.4 & 48.1 \\
\hline PI & & PL & $\mathrm{CV}$ & Satsuma & 46 & 65.2 & 32 & 31.0 & 66.1 & 48.1 \\
\hline PI & & & $\mathrm{CV}$ & Herholdt Cling & 39 & 71.7 & 11 & 25.0 & 37.5 & 48.4 \\
\hline & RK & & & BY20-11 & 48 & 62.5 & 49 & 34.6 & 89.7 & 48.6 \\
\hline & RK & & & FLA9-4 & 48 & 62.5 & 47 & 35.3 & 49.3 & 48.9 \\
\hline & RS & & & B14DR56 & 48 & 58.3 & 48 & 39.6 & 77.8 & 49.0 \\
\hline & & PL & & BY4-1050 & 37 & 81.0 & 18 & 17.0 & 38.6 & 49.0 \\
\hline NA & & & & J68-184 = Indian Cling & 43 & 44.1 & 47 & 54.1 & 79.1 & 49.1 \\
\hline NA & & & & SCRS1 & 48 & 50.0 & 83 & 50.3 & 70.1 & 50.2 \\
\hline NA & & & & SCRS10 & 39 & 76.9 & 47 & 25.0 & 93.4 & 51.0 \\
\hline & RK & & & ARK7771 = Nemaguard $*$ Siberian C & 45 & 51.1 & 0 & --- & -- & 51.1 \\
\hline NA & & & & GA107 & 41 & 51.2 & 46 & 52.1 & 83.7 & 51.7 \\
\hline & RK & & & Nemaguard & 48 & 54.1 & 49 & 49.2 & 84.2 & 51.7 \\
\hline & RK & & & B 16-167 & 42 & 66.6 & 42 & 38.1 & 91.6 & 52.4 \\
\hline & & PL & & BY7797-12 & 0 & -.- & 22 & 53.2 & 69.0 & 53.2 \\
\hline & RS & & & BI4DR53 & 40 & 67.5 & 48 & 39.5 & 81.8 & 53.5 \\
\hline NA & & & & J68-69 = Indian Blood & 43 & 48.8 & 48 & 58.2 & 85.3 & 53.5 \\
\hline & RK & & & FV3-2 = Nemaguard $*($ Nemaguard $* S-37)$ & 47 & 53.1 & 48 & 54.2 & 91.7 & 53.7 \\
\hline PI & & & $\mathrm{CV}$ & Gaschina Novembre & 45 & 57.7 & 48 & 50.0 & 76.6 & 53.9 \\
\hline PI & & & $\mathrm{CV}$ & Nooiens Herholdts & 44 & 63.6 & 33 & 44.5 & 71.0 & 54.1 \\
\hline NA & & & & J68-52 & 47 & 61.7 & 48 & 47.7 & 86.8 & 54.7 \\
\hline NA & RS & & & Tennessee Natural R27 & 49 & 59.1 & 49 & 51.0 & 85.2 & 55.1 \\
\hline PI & & & $\mathrm{CV}$ & Eagle Beak & 48 & 68.7 & 50 & 41.7 & 100 & 55.2 \\
\hline & RS & & & Lovell RL & 48 & 47.9 & 48 & 62.5 & 90.4 & 55.2 \\
\hline & & & $\mathrm{CV}$ & Redglobe & 46 & 58.6 & 48 & 52.1 & 85.3 & 55.4 \\
\hline PI & & & $\mathrm{CV}$ & Quetta & 18 & 55.6 & 0 & --- & --- & 55.6 \\
\hline PI & & .. & & P. davidiana $\mathrm{VB}$ & 28 & 85.7 & 25 & 26.7 & 100 & 56.2 \\
\hline NA & RS & & & Boone County & 24 & 66.6 & 14 & 46.0 & 67.0 & 56.3 \\
\hline PI & & & $\mathrm{CV}$ & China Flat & 40 & 80.0 & 18 & 33.3 & 53.3 & 56.7 \\
\hline PI & & & $\mathrm{CV}$ & Peregrine & 47 & 59.6 & 17 & 54.6 & 100 & 57.1 \\
\hline PI & RK & & $\mathrm{CV}$ & Shalil & 47 & 76.6 & 43 & 39.1 & 67.3 & 57.9 \\
\hline & RK & & & FLA14-11 & 44 & 70.4 & 21 & 46.0 & 60.0 & 58.2 \\
\hline & RK & & & BY3-6 & 45 & 54.3 & 48 & 62.5 & 100 & 58.4 \\
\hline PI & & & $\mathrm{CV}$ & Terzarolo Col Pizzo & 43 & 65.1 & 48 & 52.1 & 100 & 58.6 \\
\hline & RS & & & B14DR57 & 46 & 76.0 & 48 & 41.6 & 90.0 & 58.8 \\
\hline PI & $\mathrm{RS}$ & & & GF677 & 27 & 59.2 & 0 & -- & --- & 59.2 \\
\hline NA & RS & & & Ferris & 48 & 70.8 & 48 & 48.0 & 67.3 & 59.4 \\
\hline NA & RS & & & Tennessee Natural R2 & 46 & 78.2 & 48 & 41.7 & 79.7 & 60.0 \\
\hline PI & & & & Ferganensis 2446 & 48 & 79.1 & 42 & 45.1 & 90.0 & 62.1 \\
\hline PI & & & $\mathrm{CV}$ & Rogani Gow & 45 & 73.3 & 47 & 51.3 & 93.7 & 62.3 \\
\hline PI & & & & Tos China \#1 & 48 & 83.3 & 46 & 44.7 & 90.0 & 64.0 \\
\hline & RS & & & Rutgers Redleaf & 46 & 73.9 & 48 & 54.1 & 100 & 64.0 \\
\hline PI & & & $\mathrm{CV}$ & Saharan Pur 1 & 48 & 79.1 & 48 & 50.0 & 85.3 & 64.6 \\
\hline PI & & & $\mathrm{CV}$ & Saharan Pur 2 & 47 & 55.3 & 48 & 75.0 & 97.5 & 65.2 \\
\hline & RK & & & B594520-3 & 47 & 87.2 & 48 & 43.7 & 67.8 & 65.5 \\
\hline NA & RS & & & NA8 & 42 & 83.3 & 48 & 47.8 & 69.3 & 65.6 \\
\hline & RK & & & BY $49-12$ & 48 & 79.1 & 48 & 52.1 & 93.3 & 65.6 \\
\hline PI & $\mathrm{RS}$ & & & Siberian C & 51 & 90.1 & 48 & 41.7 & 100 & 65.9 \\
\hline
\end{tabular}




\begin{tabular}{|c|c|c|c|c|c|c|c|c|c|c|}
\hline & ccession & & & & & $\begin{array}{l}\text { outh } \\
\text { rolina }\end{array}$ & & $\begin{array}{r}\text { Georg } \\
\% \\
\end{array}$ & SL & $\begin{array}{c}\text { Both } \\
\text { states } \\
\end{array}$ \\
\hline NA/PI & RK/RS & PL & $\mathrm{CV}$ & Accession & $\mathbf{N}$ & $\%$ PTSL & $\mathrm{N}$ & All trees & $-\operatorname{Root} \operatorname{rot}^{\mathrm{y}}$ & $\%$ PTSL \\
\hline & RK & & & BY9-10 & 45 & 82.2 & 48 & 49.8 & 79.1 & 66.0 \\
\hline PI & RS & & & Chui Lum Tao & 48 & 79.1 & 48 & 54.1 & 100 & 66.6 \\
\hline PI & & & $\mathrm{CV}$ & Angel & 48 & 85.4 & 48 & 47.8 & 86.8 & 66.6 \\
\hline & RS & & & B14DR60 & 46 & 78.2 & 48 & 56.3 & 92.7 & 67.3 \\
\hline PI & & & $\mathrm{CV}$ & De Coosa & 47 & 65.9 & 48 & 68.7 & 100 & 67.3 \\
\hline & RK & & & BY $40-2$ & 46 & 97.8 & 48 & 37.5 & 94.5 & 67.7 \\
\hline PI & RS & & & Harrow Blood & 41 & 95.1 & 48 & 41.1 & 67.7 & 68.1 \\
\hline & RS & & & ARK7768 = Boone* Siberian C & 44 & 86.3 & 4 & 50.0 & 100 & 68.2 \\
\hline PI & & & $\mathrm{CV}$ & Violette Hative & 48 & 68.7 & 0 & --- & -- & 68.7 \\
\hline PI & & & $\mathrm{CV}$ & Sary Oiler & 47 & 89.3 & 47 & 48.7 & 90.6 & 69.0 \\
\hline & RK & & & J67-30 = Rancho Res. OP & 45 & 53.3 & 48 & 84.8 & 100 & 69.1 \\
\hline PI & & & CV & Soleil D'Octobre & 3 & 100 & 48 & 39.5 & 81.0 & 69.8 \\
\hline & RS & & & ARK7774 = Boone $*$ NJ51 10435 & 40 & 70.0 & 0 & --- & --- & 70.0 \\
\hline PI & & & & PI1 17679 & 39. & 87.1 & 48 & 56.3 & 100 & 71.7 \\
\hline NA & RS & & $\mathrm{CV}$ & J67-4 = Elberta OP & $48^{\circ}$ & 87.5 & 48 & 56.3 & 87.1 & 71.9 \\
\hline PI & & & & Shau Thai Tao & 40 & 77.5 & 48 & 66.7 & -90.6 & 72.1 \\
\hline & RK & & & BY3-12 & 47 & 87.2 & 49 & 57.8 & 97.1 & 72.5 \\
\hline & RK & & & BY9-1 & 46 & 82.6 & 48 & 62.5 & 97.1 & 72.6 \\
\hline PI & & & & Mao Tao & 38 & 89.4 & 48 & 56.1 & 82.2 & 72.8 \\
\hline & RK & PL & & Marianna 2624 cutting & 14 & 100 & 24 & 45.7 & 100 & 72.9 \\
\hline & RK & & & BY $40-12$ & 45 & 86.6 & 10 & 60.0 & 100 & 73.3 \\
\hline NA & RS & & & Stark Redleaf & 48 & 91.6 & $49^{-}$ & 56.2 & 79.7 & 73.9 \\
\hline NA & RS & & & $\mathrm{J} 68-55=$ Tennessee Natural OP & 44 & 75.0 & 48 & 73.0 & 100 & 74.0 \\
\hline PI & RS & PL & & Damas 1869 & 20 & 75.0 & 0 & --- & --- & 75.0 \\
\hline & RK & & & R76 & 47 & 95.7 & 42 & 57.1 & 100 & 76.4 \\
\hline & RS & & & RL8 & 47 & 78.7 & 50 & 74.5 & 96.8 & 76.6 \\
\hline & RK & & & BY9-13 & 48 & 79.1 & 48 & 75.0 & 90.7 & 77.1 \\
\hline & RS & & & Lovell x Nemaguard & 48 & 85.4 & 48 & 68.7 & 100 & 77.1 \\
\hline PI & & & & PI146137 & 47 & 87.2 & 49 & 67.0 & 100 & 77.1 \\
\hline & RK & & & BY3-10 & 48 & 89.6 & 48 & 66.6 & 100 & 78.1 \\
\hline & RK & & & BY20-8 & 46 & 100 & 30 & 56.6 & 100 & 78.3 \\
\hline & & & $\mathrm{CV}$ & Redglobe cutting & 38 & 84.2 & 49 & 73.0 & 96.8 & 78.6 \\
\hline & RK & & & B58RL255 & 42 & 85.7 & 48 & 73.0 & 100 & 79.4 \\
\hline PI & RS & & & Tzim Pee Tao & 48 & 85.4 & 48 & 75.0 & 100 & 80.2 \\
\hline PI & RS & & & $\mathrm{J} 68-59=P$. davidiana & 44 & 97.7 & 48 & 64.5 & 89.1 & 81.1 \\
\hline NA & & & & J68-254 = Red English & 47 & 93.6 & 48 & 68.7 & 90.0 & 81.2 \\
\hline NA & & & & J68-14 & 38 & 92.1 & 49 & 71.6 & 100 & 81.9 \\
\hline & RK & & & ARK7861 & 45 & 82.2 & 0 & --- & --- & 82.2 \\
\hline & RK & & & BY20-4 & 47 & 91.4 & 48 & 75.0 & 97.8 & 83.2 \\
\hline PI & & & $\mathrm{CV}$ & Genovese & 49 & 83.6 & 0 & -- & --- & 83.6 \\
\hline & RS & & & ARK7763 = Siberian C*Rut. RL & 31 & 83.8 & 0 & --- & --- & 83.8 \\
\hline PI & RK & & $\mathrm{CV}$ & Yunnan & 40 & 82.5 & 48 & 85.3 & 100 & 83.9 \\
\hline PI & & & $\mathrm{CV}$ & Killiekrankie & 48 & 89.6 & 48 & 79.1 & 100 & 84.4 \\
\hline & RK & & & BY49-7 & 45 & 100 & 48 & 70.7 & 100 & 85.4 \\
\hline & RK & & & ARK $7860=$ S-60*Boone & 39 & 87.1 & 0 & --- & $\cdots$ & 87.1 \\
\hline PI & & & & PI1 19840 & 48 & 87.5 & 0 & --- & --- & 87.5 \\
\hline & RS & & & ARK $7772=$ Boone*Rut. RL & 16 & 87.5 & 0 & --- & -- & 87.5 \\
\hline PI & RS & & & Montclar & 39 & 89.7 & 0 & --- & --- & 89.7 \\
\hline & & PL & $\mathrm{CV}$ & Opata & 21 & 90.5 & 0 & --- & --- & 90.5 \\
\hline PI & .. & & CV & Royal George & 18 & 94.4 & 0 & --- & --- & 94.4 \\
\hline PI & & & $\mathrm{CV}$ & Pineapple & 42 & 95.2 & 0 & --- & -- & 95.2 \\
\hline PI & & & $\mathrm{CV}$ & Bolivian Cling & 22 & 95.4 & 0 & --- & --- & 95.4 \\
\hline PI & & & $\mathrm{CV}$ & Khodjent Kostokos & 29 & 96.6 & 0 & --- & -- & 96.6 \\
\hline & RK & & & BY $40-3$ & 32 & 100 & 0 & --- & --- & 100 \\
\hline & RK & & & ARK $7762=S-60^{*}$ Rut. RL & 23 & 100 & 0 & --- & --- & 100 \\
\hline PI & & & & PI134401 & 14 & 100 & 0 & --- & -- & 100 \\
\hline & RS & & & ARK7764 = Siberian C*NJ5110435 & 14 & 100 & 0 & $\ldots$ & --- & 100 \\
\hline & RS & & & ARK7759 = Siberian C*RR62-416 & 20 & 100 & 0 & --- & $--\cdot$ & 100 \\
\hline Waller- & ancan MsI & & & & & 31.8 & & 27.0 & & 24.3 \\
\hline
\end{tabular}

${ }^{\mathrm{z}}$ Accession type: NA = natural or feral (U.S.) selection; PI = plant introduction or of foreign origin; RK = root-knot-nematode-resistant rootstock; RS = other rootstock; $\mathrm{PL}=$ plum; $\mathrm{CV}=$ fruit cultivar.

yPercentage based on limited data set includes only trees dead from PTSL or still alive at end of experiment [number of trees dead from PTSL/(initial tree total trees dead from root rot) $\times 100]$.

${ }^{\mathrm{x}}$ Minimum significant difference $(\mathrm{k}$ ratio $=100$ ) for separating means within a column.

the difficulty of budding so many trees. About 85 accessions produced enough seedlings to be fully replicated at both sites. 'Redglobe' and four standard lines were included as rooted cuttings. Commercially budded trees of 'Pekin'/Lovell from North Carolina were also added as standards. Trees were dug Jan. 1983, sorted by size for blocking purposes, and planted at the Sandhill Experiment Station, Elgin (near Columbia), S.C. (lat. $34^{\circ} 8^{\prime} \mathrm{N}$; climate zone 7B), and at the U.S. Dept. of Agriculture (USDA) facility at Byron, Ga. (lat. $32^{\circ} \mathrm{N}$; climate zone $8 \mathrm{~A}$ ). The USDA planting consisted of eight randomized blocks, each containing a six-tree plot of most accessions. Soil type was a Faceville fine sandy loam. The site had a history of PTSL and had last been in peaches 2 years earlier. Preplant ring nematode populations obtained in Nov. 1982 ranged from 50 to 360 per $150 \mathrm{~cm}^{3}$ soil. The Elgin site consisted of six randomized 
blocks of eight-tree plots. each on a site long planted to peaches. Soil type was a Lakeland fine sand. Tree spacing at both sites was $0.6 \mathrm{~m}$ within the row and 4-5 $\mathrm{m}$ between rows.

Trees were not thinned, and sprayed only on the trunk each fall for peachtree borer (Synathedon spp.). Fertilizer and herbicide application followed commercial recommendations. Trees were minimally pruned of lower limbs when young, then left unpruned, except for hedging in Fall (typically Dec.) 1985, 1986, 1988, and 1990 at Byron and Fall 1989 at Elgin. Each fall through 1986, all plots in four selected- blocks at Byron were sampled for ring nematodes. Two probes of soil per tree were obtained and combined from the inner four of six treatment trees. Nematodes were extracted from $100-\mathrm{cm}^{3}$ subsamples using elutriation (Byrd et al., 1976) combined with sugar-centrifugal flotation (Jenkins, 1964) and counted.

Survival data were tested using analysis of variance (SAS Institute, 1987). Transformation was ineffective in reducing the modest correlation between means and variances; therefore. no transformation was used. Means are based on plot means and separated using Wailer-Duncan $\mathrm{k}$ ratio test. Lines lacking substantial replication were excluded from the Wailer-Duncan test. Each spring (generally April) and fall (Byron only, 1983-86), dead trees were removed and the cause of death determined by digging around the roots. A few trees died from transplant shock, crown gall, or unknown causes. When mycelium of Armillaria tabescens (Stop.:Fr.) Dennis was seen on the trunk or roots, Clitocybe root rot was designated the cause of death. This disease is endemic in the southeastern United States and causes substantial tree losses, particularly in heavier soils. Typical symptoms of PTSL were trunk cambial browning ending at the soil line and healthy roots, often with rootstock suckers appearing from below the ground. In South Carolina, PTSL symptoms often included bacterial canker.

\section{Results and Discussion}

Tree losses due to PTSL in Georgia were highest in 1986 and 1987 (Fig. 1). Tree losses were higher in years following fall pruning (hedging). Early losses were higher in Georgia than South Carolina (Fig. 1). Correlations of nematode counts and tree death were low (Georgia data not shown; South Carolina data in Reighard et al., 1989b), probably because the sample did not accurately reflect the true population under the trees. Root rot was not a cause of tree death in South Carolina. Death from root rot at Byron was fairly constant until tree counts declined in 1990. By 1991 only $11 \%$ of the original 4885 trees survived at Byron compared to $30 \%$ of the original 5366 at Elgin in 1990.

The large variation present in mean survival (Table 1) can be attributed to environment, nematode population density, and genetic heterogeneity. The ring nematode was detected under all test lines, but populations varied across the block (Fig. 2, Table 2). The large degree of variation in the initial ringnematode population density in the test plot at Byron was attributed to tree row influence resulting from a prior planting. Higher nematode counts were detected in soil taken closest to old tree rows. Such effects must be considered when establishing a new test orchard on an old site.

Tree losses at Byron due to Clitocybe root rot confound the losses due to PTSL. However, PTSL is more common in years 3 to 6 of an orchard, whereas root rot tends to kill trees later. Some trees may have been killed by both pathogenic agents together. The second column of percentages for Georgia in Table 1 presents PTSL losses as a proportion of live trees plus trees that died from PTSL (= total trees minus root-rot losses). A large difference in the two percentages for a given line in Georgia indicates higher root-rot losses.

Some variability in tree survival within lines may relate to genotype. Recent results indicate substantial genetic variability for longevity within a line (Beckman et al., 1993). Therefore, some survivors from otherwise poorly performing lines have been propagated for further testing.

The performance of standard rootstock (means for Lovell, Halford, Nemaguard, Siberian C) closely resembles that reported for budded trees in the southeastern United States (Dozier et al., 1984; Ritchie and Clayton, 1981; Sharpe et al., 1989). Lovell and Halford survived longest, followed by Nemaguard, with Siberian C surviving the shortest period of time. Losses for all lines are shown in Table

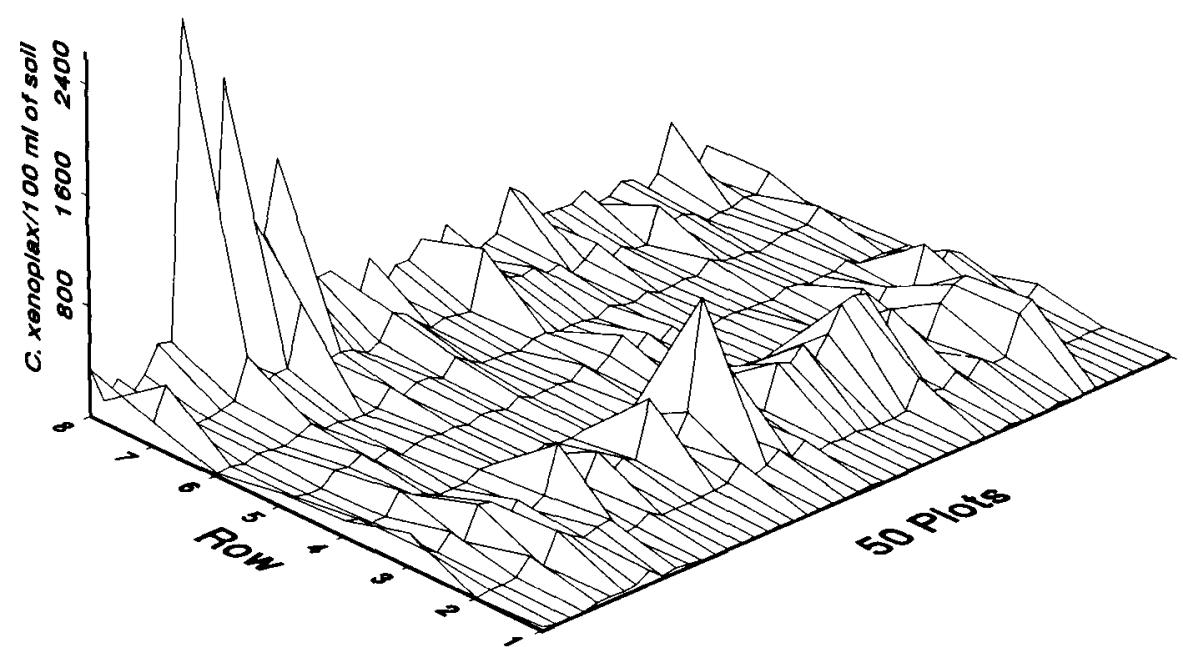

Fig. 2. Ring nematode population density beneath a portion (four blocks) of the Byron, Ga., rootstock planting that was established on a site previously planted to peach. Sampled Nov. 1983, about 10 months after planting trees. Each plot in each row was sampled ( 400 total).

Table 2. Mean (士SE) Criconemella xenoplax populations per $100 \mathrm{~cm}^{3}$ of soil under seedlings of selected peach rootstocks and overall (102 accessions) on a PTSL site in Byron, Ga., 1983-89. Means of four blocks using pooled sample from each 6-tree plot.

\begin{tabular}{lccccccc}
\hline Accession & 1983 & 1984 & 1985 & 1986 & 1987 & 1988 & 1989 \\
\hline B594520-9 & $66 \pm 23$ & $1176 \pm 242$ & $1617 \pm 879$ & $598 \pm 190$ & $213 \pm 19$ & $67 \pm 67$ & $502 \pm 248$ \\
Lovell & $39 \pm 17$ & $1559 \pm 762$ & $1255 \pm 323$ & $506 \pm 88$ & $237 \pm 49$ & $184 \pm 130$ & $105 \pm 34$ \\
Pekin/Lovell & $15 \pm 5$ & $803 \pm 432$ & $1507 \pm 497$ & $690 \pm 157$ & $180 \pm 30$ & $92 \pm 53$ & -- \\
Halford & $23 \pm 4$ & $766 \pm 102$ & $793 \pm 250$ & $1748 \pm 1442$ & $285 \pm 154$ & $184 \pm 130$ & $\cdots$ \\
Nemaguard & $85 \pm 31$ & $1564 \pm 643$ & $1071 \pm 287$ & $874 \pm 138$ & $254 \pm 47$ & $61 \pm 61$ & $311 \pm 90$ \\
Siberian C & $179 \pm 145$ & $1176 \pm 385$ & $992 \pm 361$ & 1656 & -- & $-\cdots$ & $-\cdots$ \\
Overall & $119 \pm 11$ & $1187 \pm 46$ & $1567 \pm 59$ & $1149 \pm 49$ & $306 \pm 15$ & $305 \pm 26$ & $331 \pm 83$ \\
\hline
\end{tabular}


seedlings reacted similarly to budded trees in this test. It would be more laborious and expensive to do such tests on budded trees. However, screening with unbudded trees may select for genotypes that survive well because of scion physiology, rather than because of rootstock effect.

B594520-9 was the best survivor at both sites (Table 1, Fig. 1), and sibling line B594520-8 also survived well. However, statistical superiority to Lovell was not evident until 1989, 6 years after planting. In a follow-up planting consisting of budded trees at a commercial spacing on a severe PTSL site in South Carolina (Reighard et al., 1991), BY520-9 (bulked seed of B594520-9 seedling selections) is surviving substantially better than Lovell in the 4 th year. This increased longevity is not due to C. xenoplax resistance (Table 2).

As expected, based on B594520-9's parentage, which traces back to S-37 and Nemaguard, preliminary results also indicate that B594520-9 selections exhibit resistance comparable to Nemaguard to several rootknot species. B594520-9 was a remnant of the root-knot-resistant rootstock development program carried on by the USDA and de-emphasized after the introduction of Nemaguard in 1959. The source and nature of the factors resulting in greater longevity for B594520-9 and its surviving seedlings (collectively known as BY520-9), as well as for B594520-8, are unknown, but they may have resulted from outcrossing that occurred during several cycles of open pollination. Unfortunately, the original B594520-9 selection no longer exists. BY520-9 has been released cooperatively by the USDA-Agricultural Research Service and Clemson Univ. (Okie et al., 1994).

\section{Literature Cited}

Beckman, T.G. , W.R. Okie, and A.P, Nyczepir. 1993. Use of clonally replicated seedlings in fieldscreeningfor resistance to peach tree short life. J. Amer. Soc. Hort. Sci. 118:115-118.

Byrd, D.W., Jr., et al. 1976. Two semi-automatic elutriatorsforextractingnematodes and certain fungi from soil. J. Nematol. 8:206-212.

Cain, D., E. Zehr, W. Okie, and A. Nyczepir. 1986. Screening of Prunus germplasm for resistance to Criconemella xenoplax and peach tree short life.HortScience 21:710. (Abstr.)

Dozier, WA., Jr., et al. 1984. Survival, growth, and yield of peach trees as affected by rootstock. HortScience 19:26-30.

Jenkins, W.R. 1964. A rapid centrifugal flotation method for separating nematodes from soil. Plant Dis. Rpt. 48:692.

Johnstone, F.W., Jr. 1971. A preliminary report on testing peach rootstock on the site of a "declined"peachorchard in Fort Valley. Proc. 30th Annu. Convention Natl. Peach Council.

Okie, W. R., T.G. Beckman, and A.P. Nyczepir. 1991. Prunus rootstock germplasm with increased longevity in the southeastern U.S HortScience26:710. (Abstr.)
Okie, W. R., T.G. Beckman, A.P. Nyczepir, G.L. Reighard, WC. Newall, Jr., and E.I. Zehr. 1994. BY520-9,apeachrootstockfor the southeastern United States that increases scion longevity. HortScience 29:705-706.

Okie, W. R., A.P. Nyczepir, and C.C. Reilly. 1987. Screeningofpeachand other Prunus species for resistancetoringnematodeinthe greenhouse. J. Amer. Soc. Hort. Sci. 112:67-70.

Reighard, G. L., D.W. Cain, and WC. Newall, Jr. 1989a. Relationship of chilling requirement in Prunus persica (L.) Batsch to peach tree short life. Fruit Var. J. 43:121-125.

Reighard, G. L., W.C. Newall, Jr., and D.W. Cain. 1989b. Screening Prunus germplasm for potential rootstock for South Carolina replant sites. Acta Hort. 254:287-290.

Reighard, G. L., W.C. Newall, Jr., E.I. Zehr, W.R. Okie, and T.G. Beckman.1991. Short life mortalityof 'Redhaven' and 'Springcrest' peach on selected rootstock. Proc. SE Professional Fruit Workers Conf. vol. 6.

Ritchie, D.F. and C.N. Clayton. 1981. Peach tree short life: A complex of interacting factors. Plant Dis. 65:462-469.

SASInstitute.1987. SAS/STAT guide for personal computers. Version 6 edition. SAS Inst., Cary, N.C.

Sharpe,R. R., C.C. Reilly, A.P. Nyczepir, and W.R Okie. 1989. Establishment of peach in a replant site as affected by soil fumigation, rootstock, and pruning date. Plant Dis. 73:412-4 15.

Westcott,S.W.,Ill.,andE.I, Zehr. 1991. Evaluation of host suitability in Prunus for Criconemella xenoplax. J. Nematol. 23:393-401. 